International Journal of Pure and Applied Mathematics

Volume 111 No. 1 2016, 141-147

ISSN: 1311-8080 (printed version); ISSN: 1314-3395 (on-line version)

url: http://www.ijpam.eu

doi: 10.12732/ijpam.v111i1.13

ijpam.eu

ON THE WEAK CONVERGENCE OF

A MAXIMUM LIKELIHOOD ESTIMATOR

\author{
Sofiane Grira \\ Department of Mathematics and Applied Sciences \\ College of Arts and Sciences \\ Abu Dhabi University \\ Al Ain Campus, P.O. Box 1790, Abu Dhabi, UNITED ARAB EMIRATES
}

Abstract: In this paper we study the behavior of a maximum likelihood estimator $\hat{\theta}$. Feigin (1985) had shown that if $M_{\mathrm{t}}$ is a square integrable martingale then $\frac{M_{\mathrm{t}}}{\sqrt{\left.<M_{\mathrm{t}}\right\rangle}}$ converges in distribution to a normal. We will prove a similar result for the maximum likelihood estimator. We will show that we could write $e^{\theta \mathrm{t}}\left(\hat{\theta}_{\mathrm{t}}-\theta\right)$ under the form $e^{\theta \mathrm{t}} \frac{M_{\mathrm{t}}}{\left\langle M_{\mathrm{t}}\right\rangle}$ which converges weakly to a Cauchy distribution.

AMS Subject Classification: 60F05, 60G44

Key Words: stochastic differential equation, martingale, maximum likelihood estimator

\title{
1. Preliminaries and Notations
}

Let $\Omega$ be the set of continuous functions $\omega: \mathbb{R}^{+} \rightarrow \mathbb{R}$. And let's define a filtration on $\Omega$ by

$$
\mathcal{F}_{t}=\cap_{s>t} \sigma(\omega(u): u \leq s)
$$

We define $\mathcal{F}$ as the smallest $\sigma$-algebra containing $\mathcal{F}_{t} \cdot\left(\Omega, \mathcal{F}, \mathcal{F}_{t}\right)$ is called the canonical space. Let $\left(\Omega^{\prime}, \mathcal{F}^{\prime}, \mathcal{F}_{t}^{\prime}, P^{\prime}\right)$ be the basic probability space. Let $\Theta$ be

$\begin{array}{lr}\text { Received: } & \text { October 26, } 2016 \\ \text { Revised: } & \text { November 11, } 2016 \\ \text { Published: } & \text { December 6, } 2016\end{array}$

(C) 2016 Academic Publications, Ltd.

url: www.acadpubl.eu 
a subset of $\mathbb{R}^{n}$ with a non-empty interior. Suppose that for all $\theta \in \Theta$ the stochastic differential equation:

$$
d Y_{t}=\beta\left(\theta ; t, Y_{t}\right) d t+\gamma\left(t, Y_{t}\right) d W_{t}, \quad Y_{0}=x_{0}
$$

has a solution $Y^{\theta} \cdot x_{0}$ is a $d$-dimensional vector and

$$
\begin{aligned}
& \gamma: \mathbb{R}^{+} \times \mathbb{R}^{d} \rightarrow \mathbb{R}^{d} \times \mathbb{R}^{m} \\
& \beta(\theta, . . .): \mathbb{R}^{+} \times \mathbb{R}^{d} \rightarrow \mathbb{R}^{d}
\end{aligned}
$$

are Borel functions.

The solution $Y^{\theta}$ induces a probability measure $P_{\theta}$ on $(\Omega, \mathcal{F})$.

Let $c(t, x)=\gamma(t, x) \gamma(t, x)^{T}$.

Definition 1.1. A strong solution of the stochastic differential equation (1) is a process $Y=\left\{Y_{t} ; 0 \leq t<\infty\right\}$ continuous with the following properties:

(i) $Y$ is adapted to the filtration $\mathcal{F}_{t}$,

(ii) $P\left(X_{0}=\xi\right)=1$,

(iii) $P\left(\int_{0}^{t}\left(\left|\beta\left(\theta ; s, Y_{s}\right)\right|+\gamma^{2}\left(s, Y_{s}\right)\right) d s<\infty\right)=1$ holds for every $1 \leq i \leq d$,

(iv) (1) holds almost surely.

Theorem 1.2. Suppose that for every $n \geq 1$ there exists a constant $K_{n}>0$ such that for every $t \geq 0,\|x\| \leq n$ and $\|y\| \leq n$ :

$$
\|\beta(t, x)-\beta(t, y)\|+\|\gamma(t, x)-\gamma(t, y)\| \leq K_{n}(\|x\|-\|y\|) .
$$

Then strong uniqueness holds for equation (1).

Proof. See [7].

We define for each $\theta \in \Theta$ a $d$-dimensional process $y(\theta)$ by:

$$
y_{t}(\theta)=\beta\left(\theta, t, Y_{t}\right)-\beta\left(\theta_{0}, t, Y_{t}\right)
$$

where $\theta_{0} \in \Theta$ is fixed, and another process $\alpha_{t}$ defined by

$$
\alpha_{t}(\theta)=c_{t}^{-1} y_{t}(\theta)
$$

where $c_{t}=c\left(t, Y_{t}\right)$. Finally let's set

$$
A_{t}(\theta)=\int_{0}^{t} \alpha_{s}(\theta)^{T} c_{s} \alpha_{s}(\theta) d s .
$$


Now we can express a Theorem which will give us the expression of the likelihood function

$$
L_{t}(\theta)=\left.\frac{d P_{\theta}}{d P_{\theta_{0}}}\right|_{\mathcal{F}_{\mathrm{t}}}=\frac{d P_{\theta}^{t}}{d P_{\theta_{0}}^{t}}
$$

where $d P_{\theta}^{t}=\left.P_{\theta}\right|_{\mathcal{F}_{\mathrm{t}}}$.

Theorem 1.3. Suppose that

$$
P_{\theta}^{t}\left(A_{t}(\theta)<\infty\right)=P_{\theta_{0}}^{t}\left(A_{t}(\theta)<\infty\right)=1
$$

Then

$$
P_{\theta}^{t} \sim P_{\theta^{(0)}}^{t}
$$

and

$$
\frac{d P_{\theta}^{t}}{d P_{\theta^{(0)}}^{t}}=\exp \left\{\int_{0}^{t} y_{s}(\theta)^{T} c_{s}^{-1} d Y_{s}^{c}\left(\theta^{(0)}\right)-\frac{1}{2} \int_{0}^{t} y_{s}(\theta)^{T} c_{s}^{-1} y_{s}(\theta) d s\right\}
$$

where

$$
Y_{s}^{c}\left(\theta^{(0)}\right)=Y_{t}-x_{0}-\int_{0}^{t} b\left(\theta ; s, Y_{s}\right) d s
$$

Proof. See [9].

Theorem 1.4. Let $X_{n}$ be a submartingale. Suppose it exists $p>1$ such that $\sup E\left[\left|X_{n}\right|^{p}\right]<\infty$, then it exists an integrable random variable $X_{\infty}$ such that

$$
X_{n} \underset{n \rightarrow \infty}{\longrightarrow} X_{\infty} \text { a.s. }
$$

and

$$
X_{n} \underset{n \rightarrow \infty}{\stackrel{L^{1}}{\longrightarrow}} X_{\infty}
$$

Proof. See [8].

\section{Main Result}

Theorem 2.1. Let's suppose that the space $(\Omega, \mathcal{F}, P)$ is the classical case of $\Omega=\mathcal{C}([0,1]: \mathbb{R})$ on which we know that we can build an arbitrary number of independent Brownian movements.

Let's consider the set of one-dimensional Gaussian diffusions solutions of the differential stochastic equation :

$$
d X_{t}=\theta X_{t} d t+d W_{t}, \quad X_{0}=0,
$$


where $\theta>0$ and $W$ is a standard, one-dimensional Brownian motion. Then it exists an integrable random variable $H_{\infty}$ and a normal distribution $Z$ independent of $H_{\infty}$ such that $e^{\theta t}\left(\hat{\theta}_{t}-\theta\right)$ converges in distribution to $Z / H_{\infty}$ which is distributed according to a Cauchy law.

Proof. We have

$$
d\left(e^{-\theta s} X_{s}\right)=e^{-\theta s}\left(d X_{s}-\theta X_{s} d s\right)=e^{-\theta s} d W_{s}
$$

by integrating between 0 and $t$ we will have

$$
e^{-\theta t} X_{t}=\int_{0}^{t} e^{-\theta s} d W_{s}
$$

therefore the solution of the equation (2) is

$$
X_{t}=\int_{0}^{t} e^{\theta(t-s)} d W_{s}
$$

According to Theorem 1.2 this solution is unique. This unique solution induces a probability measure $P_{\theta}$ (obviously unique ) on $(\Omega, \mathcal{F})$ that is $P_{\theta}$ is the image of $P^{\prime}$ by the application $\omega^{\prime} \in \Omega^{\prime} \rightarrow X\left(\omega^{\prime}\right) \in \Omega$.

Since $X_{t}$ is a continuous process (because $\mathrm{W}$ is continuous) therefore

$$
A_{t}=\int_{0}^{t} X_{s}^{2} d s<\infty \quad \forall t>0 .
$$

Then by Theorem 1.3 by taking $\theta^{(0)}=0$ the likelihood function is

$$
L_{t}(\theta)=\frac{d P_{\theta}^{t}}{d P_{\theta^{(0)}}^{t}}=e^{\left(N_{\mathrm{t}} \theta-\frac{1}{2} \theta^{2} I_{\mathrm{t}}\right)}
$$

where

$$
N_{t}=\int_{0}^{t} X_{s} d X_{s}
$$

and

$$
I_{t}=\int_{0}^{t} X_{s}^{2} d s
$$

Let's calculate the maximum likelihood estimator $\hat{\theta}$.

$$
\frac{\partial}{\partial \theta} \log \left(L_{t}(\theta)\right)=N_{t}-\theta I_{t}
$$


$\Longrightarrow$

$$
\hat{\theta}_{t}=I_{t}^{-1} N_{t}
$$

On the other hand, let's set

$$
H_{t}=e^{-\theta t} X_{t}=\int_{0}^{t} e^{-\theta s} d W_{s}
$$

according to (3). But

$$
E\left[H_{t}^{2}\right]=\int_{0}^{t} e^{-2 \theta s} d s=\frac{1-e^{-2 \theta t}}{2 \theta} \leq \frac{1}{2 \theta}<\infty
$$

which imply according to Theorem 1.4 that it exists $H_{\infty}$ such that

$$
H_{t}=e^{-\theta t} X_{t} \underset{t \rightarrow \infty}{\longrightarrow} H_{\infty} \quad \text { p.s. }
$$

Moreover $H_{\infty}$ is distributed according to a normal distribution $N\left(0, \frac{1}{2 \theta}\right)$. Let's apply Itô's formula for $X_{t}$ with $f(x)=\frac{x^{2}}{2}$, we shall have

$$
\frac{X_{t}^{2}}{2}=N_{t}+\frac{t}{2}
$$

which imply

$$
N_{t}=\frac{1}{2}\left(e^{2 \theta t} H_{t}^{2}-t\right)
$$

Therefore

$$
\begin{aligned}
e^{\theta t}\left(\hat{\theta}_{t}-\theta\right)=e^{\theta t}\left(\frac{N_{t}}{I_{t}}-\theta\right) & =e^{\theta t}\left(\frac{\frac{1}{2} e^{2 \theta t} H_{t}^{2}-\frac{t}{2}}{I_{t}}-\theta\right) \\
& =\frac{e^{\theta t}}{2 I_{t}}\left(e^{2 \theta t} H_{t}^{2}-t-2 \theta I_{t}\right)
\end{aligned}
$$

But

$$
I_{t}=\int_{0}^{t} e^{2 \theta s} H_{s}^{2} d s \sim \frac{e^{2 \theta t}}{2 \theta} H_{t}^{2} \sim \frac{e^{2 \theta t}}{2 \theta} H_{\infty}^{2}
$$

which imply

$$
e^{\theta t}\left(\hat{\theta}_{t}-\theta\right) \sim \frac{\theta e^{-\theta t}}{H_{t}^{2}}\left(e^{2 \theta t} H_{t}^{2}-t-2 \theta \int_{0}^{t} e^{2 \theta s} H_{s}^{2} d s\right) .
$$


On the other hand

$$
\begin{aligned}
\int_{0}^{t} e^{2 \theta s} H_{s}^{2} d s= & \int_{0}^{t} e^{2 \theta s}\left(H_{s}-H_{t}+H_{t}\right)^{2} d s \\
= & \int_{0}^{t} e^{2 \theta s}\left(H_{s}-H_{t}\right)^{2} d s+H_{t}^{2} \int_{0}^{t} e^{2 \theta s} d s \\
& +2 H_{t} \int_{0}^{t} e^{2 \theta s}\left(H_{s}-H_{t}\right) d s .
\end{aligned}
$$

But

$$
\int_{0}^{t} e^{2 \theta s} d s=\frac{e^{2 \theta t}-1}{2 \theta} \sim e^{2 \theta t} /(2 \theta)
$$

and

$$
E\left\{\int_{0}^{t} e^{2 \theta s}\left(H_{s}-H_{t}\right)^{2} d s\right\} \sim \frac{t}{2 \theta} .
$$

Hence

$$
e^{\theta t}\left(\hat{\theta}_{t}-\theta\right) \sim \frac{4 \theta^{2} e^{-\theta t}}{H_{t}} \int_{0}^{t} e^{2 \theta s}\left(H_{t}-H_{s}\right) d s
$$

as $H_{t}-H_{s}$ is distributed according to a normal distribution, then

$$
4 \theta^{2} e^{-\theta t} \int_{0}^{t} e^{2 \theta s}\left(H_{t}-H_{s}\right) d s
$$

converges in distribution to $Z$, distributed according to a normal distribution with zero mean and variance $2 \theta^{2}$, because

$$
\begin{aligned}
\sigma_{t}^{2} & =\operatorname{Var}\left(4 \theta^{2} e^{-\theta t} \int_{0}^{t} e^{2 \theta s}\left(H_{t}-H_{s}\right) d s\right) \\
& =16 \theta^{4} e^{-2 \theta t} \int_{0}^{t} \int_{0}^{t} e^{2 \theta s} e^{2 \theta s^{\prime}} \int_{\max \left(s, s^{\prime}\right)}^{t} e^{-2 \theta u} d u d s d s^{\prime} \\
& =8 \theta^{3} e^{-2 \theta t} \int_{0}^{t} \int_{0}^{t} e^{2 \theta\left(s+s^{\prime}\right)}\left(e^{-2 \theta \max \left(s, s^{\prime}\right)}-e^{-2 \theta t}\right) d s d s^{\prime} \\
& =16 \theta^{3} e^{-2 \theta t} \int_{0}^{t} \int_{0}^{s} e^{2 \theta s^{\prime}}\left(1-e^{-2 \theta(t-s)}\right) d s^{\prime} d s \\
& =8 \theta^{2} \int_{0}^{t}\left(1-e^{-2 \theta s}\right)\left(e^{-2 \theta s}-e^{-2 \theta t}\right) d s \\
& \rightarrow 2 \theta .
\end{aligned}
$$

Finally, 


$$
\begin{aligned}
\operatorname{Cov}\left(H_{t}, 4 \theta^{2} e^{-\theta t} \int_{0}^{t} e^{2 \theta s}\left(H_{t}-H_{s}\right) d s\right) & =4 \theta^{2} e^{-\theta t} \int_{0}^{t} e^{2 \theta s} \int_{s}^{t} e^{-2 \theta u} d u d s \\
& =2 \theta e^{-\theta t} \int_{0}^{t}\left(1-e^{-2 \theta s}\right) d s \\
& \rightarrow 0 .
\end{aligned}
$$

As $H_{t}$ converges almost surely to $H_{\infty}$ and $Z$ is independent of $H_{\infty}$, then $e^{\theta t}\left(\hat{\theta}_{t}-\right.$ $\theta$ ) converges in distribution to $Z / H_{\infty}$ which is distributed according to Cauchy distribution with density function

$$
f(x)=\frac{1}{\pi} \frac{2 \theta}{1+4 \theta^{2} x^{2}} .
$$

\section{References}

[1] B.M. Brown, Martingale central limit theorems, Ann. Math. Statist, 42 (1971), 59-66, doi: $10.1214 /$ aoms/1177693494.

[2] D.J. Daley, D. Vere-Jones, An introduction to the theory of point process, Springer-Verlag, (1988), doi: 10.1137/1032025.

[3] P.D Feigin, Stable convergence of semimartingales, Stoch. Proc. App, 19 No 1 (1985), 125-134, doi: 10.1016/0304-4149(85)90044-4.

[4] P. Hall, C.C. Heyde, Martingale limit theory and its application, Academic Press, Inc., Harcourt Brace Jovanovich, Publishers, New York-London (1980). MR 624435

[5] N. Ikeda, S. Watanabe, Stochastic differential equations and diffusion processes, NorthHolland Publishing Company, (1981), doi: 10.1002/bimj.4710280425.

[6] J. Jacod, A.N. Shiryaev, Limit theorems for stochastics process, Springer-Verlag, (1987),doi: 10.1007/978-3-662-05265-5.

[7] I.Karatzas, E.S. Shreve, Brownian motion and Stochastic calculus, Springer-Verlag, (1988), doi: 10.1007/978-1-4684-0302-2.

[8] A.N. Shiryayev, Probability, Springer-Verlag, (1984), doi: 10.1007/978-1-4899-0018-0.

[9] M. Sorensen, Likelihood methods for diffusions with jumps, Prob. Pure. Applied, 6 (1991), 67-105. 
